\title{
Investigación en la práctica docente universitaria: obstáculos epistemológicos y alternativas desde la Didáctica General Constructivista
}

\author{
Julio César Tovar-Gálvez \\ Germán Antonio García Contreras \\ Universidad Antonio Nariño (Bogotá-Colombia)
}

\section{Resumen}

El artículo se estructura señalando la docencia universitaria como un amplio campo de investigación y definiendo la práctica docente como objeto de estudio. De esta manera, emerge el problema teórico y metodológico de qué y cómo observar la dimensión didáctica en la práctica docente, frente a lo cual se hace una discusión en torno a la Didáctica General como derivada de las Teorías del Aprendizaje y las Didácticas Específicas fundamentadas en la especificidad epistemológica de cada disciplina. Frente a la dificultad de encontrar didácticas específicas de todas las disciplinas que pueden hacer parte de la formación de diferentes profesionales, las cuales estén argumentadas filosófica, epistemológica, teórica y metodológicamente, se propone optar la Didáctica General Constructivista como alternativa teórica y metodológica que fundamente la investigación sobre lo didáctico de la práctica docente. El artículo continúa con una revisión amplia sobre los paradigmas Positivista y Constructivista y las didácticas que desde cada uno se sustentan, para cerrar proponiendo los elementos y procesos que caracterizan la Didáctica General Constructivista.

\section{Palabras clave}

Práctica docente - Didáctica - Epistemología - Constructivismo

- Enseñanza-aprendizaje.

\section{Contacto:}

Julio César Tovar-Gálvez

Universidad Antonio Nariño

Facultad de Educación

Bogotá-Colombia

julitovar@uan.edu.co 


\title{
An investigation into the teaching practice at the university: epistemological obstacles and alternatives based on the General Constructivist Didactics
}

\author{
Julio César Tovar-Gálvez \\ Germán Antonio García Contreras \\ Universidad Antonio Nariño (Bogotá-Colombia)
}

\begin{abstract}
The article is structured to show university teaching as a wide field of investigation, and to define the teaching practice as an object of study. In this way, the theoretical and methodological problem emerges as to what and how to observe the didactic dimension of the teaching practice, carrying out a discussion about General Didactics derived from the Theories of Learning and Specific Didactics founded on the epistemological specificity of each discipline. Faced with the difficulty of finding specific didactics for all disciplines that can be part of the formation of different professionals, which should be philosophically, epistemologically, theoretically and methodologically grounded, we propose to choose the General Constructivist Didactics as a theoretical and methodological alternative to fundament the investigation about the didactic aspect of the teaching practice. The article proceeds to a wide survey of the Positivist and Constructivist paradigms and of the didactics supported by each one of them, closing with a proposal for the elements and processes that characterize the General Constructivist Didactics.
\end{abstract}

\section{Keywords}

Teaching practice - Didactics - Epistemology - Constructivism - Teaching-learning.

Contact:

Julio César Tovar-Gálvez

Universidad Antonio Nariño

Facultad de Educación

Bogotá-Colombia

julitovar@uan.edu.co 
La docencia universitaria se constituye como un problema de investigación, en tanto la universidad, la sociedad y la cultura están en constante cambio, exigiendo formadores de profesionales que respondan a contextos cambiantes, acelerados y con demandas diversas; es así como la universidad, los docentes universitarios y los profesionales en formación inicial están enfrentándose a los cambios económicos y políticos, al desarrollo científico y tecnológico en un mundo de producción y desigualdades (HERNÁNDEZ, 2002; FRANCIS, 2006).

Varios aspectos generales de la investigación en formación y práctica docente, también son interés en la investigación de la docencia universitaria; por ejemplo, en términos de Figueroa y Páez (2008), son relevantes los temas relacionados con el pensamiento del docente, tales como: las concepciones, teorías implícitas, creencias, lenguaje en el aula, acciones didácticas, así como muchos otros elementos que forman parte de la dinámica educativa $\mathrm{y}$ en especial del aula. Todo ello supone que la comprensión del rol del docente universitario se debe hacer desde la multidimensionalidad y situándolo en contextos sociales y momentos específicos; es desde esta perspectiva que es posible enfocar la investigación, así como la formación de docentes con perfiles que les permitan transformar los escenarios en que se desarrollan (FRANCIS, 2006; CEBRIÁN; VAIN, 2008).

Los aspectos enunciados anteriormente son de interés para pensar el tema de la docencia universitaria, pero sobre todo es relevante preguntarse sobre el hecho que los docentes universitarios, en su mayoría, son profesionales y especialistas en su área del conocimiento específica, pero no en la educación y no en la didáctica del conocimiento de su profesión. Sobre esta base se sustenta en parte el interés en torno a la formación permanente, profesionalización, perfeccionamiento y/o excelencia de los docentes universitarios; aspecto que motiva el desarrollo de la investigación "El docente, un sujeto en construcción”, en la Universidad
Antonio Nariño, desde la cual se derivan la reflexión y propuestas particulares que se presentan en este artículo.

\section{Problema general de la formación docente universitaria}

Es muy común encontrar en la universidad de hoy docentes que son profesionales que conocen su disciplina pero, que no necesariamente aprendieron como enseñarla (MONTENEGR0; FUENTEALBA, 2010) y aunque para un gran número de profesores estas situaciones no tienen mayores implicaciones en el contexto educativo, diferentes estudios han concluido que la enseñanza de una disciplina específica se sustenta en el dominio de un conjunto de conocimientos (pedagógicos, curriculares, de los estudiantes, del contexto y del contenido que se enseña, entre otros) por lo que para enseñar una temática específica no basta el conocimiento disciplinar (SHULMAN, 1987; 1999).

La ausencia de prácticas y políticas educativas que orienten la práctica docente en la formación de profesionales en la actualidad, ha promovido que en el interior de las aulas de clases universitarias prevalezcan aún métodos educativos tradicionales en los que se privilegia la memorización de contenidos, el aprendizaje individual y la reproducción en la evaluación de los aprendizajes desarrollados por los estudiantes (OCDE, 2009), y aunque esta conclusión surge de la actualidad en educación superior en Chile, este comportamiento no es muy diferente para resto de Latinoamérica.

Respecto a este problema, es notable el avance logrado en Brasil en este campo de investigación, pues es posible identificar numerosos artículos en los que no solo se reflexiona, sino que también se estudia la práctica docente como tal, se estudian los programas de formación de docentes universitarios y se han concretado propuestas teórico-metodológicas. Una breve revisión, permite definir el complejo problema desde diversos frentes: 
- Las dinámicas en las universidades, pues la vinculación de profesores se hace teniendo como principal criterio la formación especializada en una disciplina y no en la formación pedagógica; lo que se traduce en que la docencia universitaria no es tomada como una profesión, sino como algo práctico que se sustenta en metodologías, técnicas o métodos adecuados (PRETTO; ROCHA, 2010). Así mismo, tal dinámica conlleva a que la docencia universitaria se centre en la enseñanza de las disciplinas, dejando de lado procesos como la formación por competencias y en las tecnologías de la información y la comunicación (MONTERO, 2007).

- Respecto a la forma en que los docentes orientan su práctica, CHAMLIAN, (2003) identifica que: a) unos imitan a sus propios maestros, b) otros han sido instruidos por sus colegas, c) otros hacen cursos extracurriculares, d) otros pocos se iniciaron como docentes voluntarios, en aulas prácticas o en seminarios.

- Existen falencias frente a contextos pues : a) los docentes no están formados para atender, por ejemplo, estudiantes con Necesidades Educativas Especiales, lo que influye en el fracaso o deserción (REIS; EUFRASIO; BAZON, 2010), b) la docencia universitaria, por lo general, no considera aspectos particulares de los cursos, los estudiantes y los contenidos, olvidando que la educación superior busca también formar sujetos, que sean críticos y capaces de construir (OLIVEIRA; VASCONCELLOS, 2011).

Así mismo, en los avances en cuanto a la profesionalización de los docentes universitarios en Brasil, así como en otros referentes, es posible identificar algunos principios esenciales:

- La docencia debe estar fundamentada en la relación enseñanza-investigación; es decir que el docente universitario debe proponerse la investigación en el aula
(CHAMLIAN, 2003; AGUIAR; MELO, 2005; OLIVEIRA; VASCONCELLOS, 2011).

- La práctica docente misma es el insumo para reflexionar sobre cómo orientar los procesos de formación de docentes universitarios (RIOLFI; ALAMINOS, 2007).

- Es necesario que en la universidad se rompan con las tradicionales clases academicistas que se caracterizan por delimitar la realidad a los campos de conocimiento y por estar aisladas de los contextos (AGUIAR; MELO, 2005).

\section{Investigación sobre la práctica docente universitaria}

La investigación El docente, un sujeto en construcción, tiene como objetivo hacer un diagnóstico del docente de la Universidad Antonio Nariño y de su práctica en el aula, de tal manera que se tenga una aproximación respecto a aspectos de su personalidad, las representaciones sociales de su rol y su motivación, así como respecto a las dimensiones relativas a currículo, didáctica, evaluación y clima laboral. El conocimiento de tales elementos que constituyen al docente universitario, permite comprender cuáles son las condiciones y necesidades de la comunidad, para desde allí establecer los criterios para estructurar el Centro de Excelencia Docente de la Universidad Antonio Nariño.

Para tal meta, se conforma un grupo de investigación con docentes de la Facultad de Administración de Empresas, Facultad de Psicología y Facultad de Educación; de tal manera que el docente y la práctica docente sean interpretados desde diversas perspectivas. Así, se seleccionan cien profesores de las Facultades de Psicología, Educación, Medicina y Administración de Empresas, quienes están vinculados con la Universidad desde hace más de un año, y quienes son invitados a participar en: a) Prueba de Personalidad: Perfil e Inventario de la Personalidad de Gordon (P.I.P.G.), b) Prueba de Motivación Psicosocial (MPS): José Luiz Fernández-Seara, 
y c) Grupos focales, para profundizar en sus representaciones. Posteriormente, de esta cantidad de profesores se seleccionan a 45 , con quienes se realiza: a) observación directa de tres de sus clases (registro de diario de campo), y b) entrevista a profundidad.

\section{Problema: el obstáculo epistemológico}

Para el caso particular de la observación de las clases y el análisis de la práctica docente universitaria, surge la reflexión respecto a los posibles obstáculos epistemológicos emergentes, puesto que: a) quién observa, aunque sea investigador educativo, no necesariamente tiene formación en las disciplinas particulares de las clases a observar, y b) no existen didácticas específicas o referentes particulares sobre cómo observar la práctica docente de todas las disciplinas (aunque las didácticas específicas de las ciencias naturales, de las ciencias sociales, de las matemáticas y de las lenguas están sustentadas, no existen didácticas consolidadas para la enseñanza de disciplinas como la gerencia, la administración, el mercadeo, la cirugía, la optometría, la arquitectura, entre otros).

Así, para abordar tal objetivo que permite comprender parte del complejo mapa que podría dar cuenta de la docencia universitaria y con ello aportar a solucionar problemas en cuanto a la formación permanente, perfeccionamiento o cualificación de los formadores de profesionales, es necesario preguntarse ¿qué es lo que se quiere observar de la práctica docente?, frente a lo cual, este artículo se enfoca en lo didáctico, categoría que motiva a preguntar ¿qué es lo didáctico? y ¿Qué permite evidenciar lo didáctico en la práctica docente?

\section{Desarrollo}

\section{Aspectos generales: sobre la práctica docente y lo didáctico}

En términos generales se puede asumir que lo didáctico centra su reflexión en torno al fenómeno de enseñanza-aprendizaje y las variables relacionadas. En este sentido es importante cuestionar sobre el aprendizaje, sobre los sujetos, sobre los contextos, sobre las normativas, sobre las disciplinas, entre varios aspectos, y a partir de todo ello, cómo enseñar.

Sin embargo, es necesario notar que lo didáctico como parte de todo el proceso educativo no se puede diferenciar o parcelar; es decir, no puede reducirse lo didáctico a una serie de acciones, técnicas, protocolos, estrategias o herramientas descontextualizadas de los fundamentos filosóficos, epistemológicos, pedagógicos, curriculares y evaluativos que soportan los procesos educativos.

Ante la dificultad para observar aquello que corresponde a lo didáctico en la compleja acción del docente $\mathrm{y}$, sobre todo, teniendo en cuenta la dificultad que representa para el observador el conocimiento de las didácticas específicas de cada área disciplinar, por demás reconocido como un problema a resolver en la investigación didáctica, se propone asumir: a) la existencia de una didáctica general que emerge de las teorías del aprendizaje, y la existencia de didácticas específicas que responden a la epistemología de cada disciplina, b) la conveniencia de asumir una posible didáctica general como referente universal para las observaciones de las acciones docentes de cualquier disciplina, decisión coherente con los objetivos de esta investigación, y d) asumir el constructivismo como referente epistemológico, psicológico, pedagógico y didáctico, en tanto cuenta con un amplio reconocimiento por las comunidades de académicos por su importante aporte a las nuevas perspectivas en educación en general y en tanto aporta teorías del aprendizaje que pueden orientar una didáctica general. Estos elementos enunciados son discutidos y sustentados en los apartes que se encuentran a continuación.

\section{Didáctica general y didáctica específica}

Haciendo una reflexión frente a las aparentes diferencias entre las estrategias de 
aprendizaje y las estrategias de enseñanza, Tovar-Gálvez (2008) reconoce que ambas posibilidades se fundamentan en posturas que distan en cuanto a objeto de estudio y en cuanto a soporte teórico-metodológico; sin embargo plantea la necesidad de integrarlas. De esta manera el autor argumenta la existencia de una Didáctica General sustentada en las Teorías del Aprendizaje y que por ende se enfoca en los procesos cognitivos del sujeto $\mathrm{y}$ en lograr su desarrollo a través de Estrategias de Aprendizaje; y así mismo argumenta la existencia de Didácticas Específicas, cuyos soportes principales están en la Historia y en la Epistemología Específicas de las Disciplinas, por lo que buscan establecer Estrategias de Enseñanza de los contenidos disciplinares (indistintamente del modelo epistémico).

En esta medida, hablar de Didáctica General permite ubicarse en principios que pretenden orientar de manera universal todos los procesos de aprendizaje, mientras que las Teorías del Aprendizaje buscan dar cuenta de la forma en que todos los sujetos aprenden; sin embargo, los aspectos particulares de la propuesta aparecen en la medida en que los constructos de dichas teorías varían según el sujeto, por lo que desde ello, las Estrategias de Aprendizaje tienen elementos que aportan a lo personal en el proceso.

Por otro lado, hablar de Didáctica Específica es ubicarse en la asunción que las disciplinas, campos de conocimiento o formas de saber, tienen naturaleza, estructuras y dinámicas particulares; aspecto que se puede argumentar a través de los estudios históricos y epistemológicos de cada disciplina. En esta medida, se hace necesario realizar reflexiones profundas sobre las posturas respecto al conocimiento y cómo ello conlleva a pensar en una u otra forma de enseñar. De esta manera, las Estrategias de Enseñanza se hacen específicas a las disciplinas, pero se hacen generales para los sujetos, en la medida en que las comunidades de especialistas asuman una $\mathrm{u}$ otra postura histórico-epistemológica sobre el cuerpo de conocimientos, siendo así válidas o no unas estrategias por su coherencia con dicha postura.

La discusión sobre la especificidad de las didácticas de las disciplinas ha avanzado fuertemente; por ejemplo, en el campo de la Didáctica de las Ciencias, puesto que sobre ésta se ha argumentado su Estatuto Científico, sustentado en el reconocimiento de que sus referentes teóricos y prácticos están principalmente fundamentados en los estudios histórico-epistemológicos de las ciencias, y sustentado en el hecho de que posee un carácter autónomo respecto a otros campos como la pedagogía, la psicología y la sociología; elementos que le conceden una estructura disciplinar (CACHAPUZ et al., 2001; ADÚRIZ-BRAVO; IZQUIERDO, 2002; GALLEGOBADILLO, 2004; TOVAR-GÁLVEZ, 2008, 2009).

En el sentido de lo anterior, por ejemplo, Adúriz-Bravo e Izquierdo (2002) señalan la diferencia entre los modelos didácticos (con fundamento histórico, filosófico y sociológico de las disciplinas que enseñan) y los modelos de la piscología cognitiva (centrados en teorías del aprendizaje); señalando la diferencia y por ende la independencia y especificidad de la Didáctica de las Ciencias, sin negar los aportes de la psicología cognitiva. Otros avances para sustentar las Didácticas Específicas se están logrando en: a) Didáctica de las Ciencias Sociales (AISENBERG, 1994; PAGÉS, 1994; PRATS, 2000 , 2003), b) Didáctica de las Lenguas (DÍAZ, 1996; VEZ, 2004; TRUJILLO, 2005; ZANÓN, 2007; NÚÑEZ; FERNÁNDEZ; ROMERO, 2008) y c) Didáctica de las Matemáticas (FERNÁNDEZ, 2000; ZAMBRANO, 2004; MORENO, 2007; GODINO, 2010; 2011).

Los objetivos de esta investigación, entre varios aspectos, orientan a observar sistemáticamente la dimensión didáctica de la práctica de docentes de los programas curriculares de Medicina, Administración, Psicología y de la Facultad de Educación (con programas de formación de docentes en Lengua Castellana e Inglés, Química y Educación Ambiental, Matemáticas, Ciencias Sociales y Básica 
Primaria). Con este contexto se reconoce la limitación para el observador, en tanto la diversidad de Didácticas Específicas, emergentes de las epistemologías de las múltiples disciplinas que conforman los programas curriculares enunciados; lo que conlleva a considerar como alternativa recurrir a una Didáctica General, para este caso constructivista, como fundamento para dicha observación.

\section{Las grandes tradiciones didácticas emergentes de las teorías del aprendizaje}

\section{a. Postura Tradicional (empirismo-positivismo y conductismo):}

Parte de la historia del paradigma se basa en el prestigio que adquieren las ciencias con el modelo mecánico de Newton, lo que significa nuevas miradas sobre lo que es $\mathrm{y}$ lo que no es científico. Ya desde 1602 Bacon (apud MENA; SALVATICO, 2002) hace su trabajo en el que determina la producción del conocimiento a través de métodos empíricos y de manera inductiva, suponiendo que quien investiga está en busca de descubrir las leyes de la naturaleza, por demás una verdad a la que se llega de manera objetiva. Sin embargo, Comte (apud TISKI, 2010) difiere en cuanto a la forma de construir conocimiento $\mathrm{y}$, aunque se ubica en la realidad ontológica, postula una lógica hipotético-deductiva. Tales posturas son trasladadas al campo de las humanidades y sociales, en busca de la rigurosidad científica; por tal, surge la Sociología (DURKHEIM, 1978) como la ciencia que de manera rigurosa estudia los hechos sociales, considerando los sujetos y los grupos como cosas u objetos manipulables y susceptibles de estudio por medio del método científico.

En esta misma lógica, el campo psicológico, cuya historia se ubica en las reflexiones realizadas por los griegos respecto al alma o lo intangible, entra en una era cientificista, al tratar de considerar la estructura de la física clásica y con ello hacer tangible y medible su objeto de estudio; proceso con el que surge lo que más adelante se llamaría el Conductismo. La relación entre la psicología del aprendizaje y el empirismo-positivismo ya ha sido señalada antes por Pozo (1989), para quien una de las principales tesis del paradigma es que "el conocimiento es una copia de la realidad, el cual es simplemente acumulado por simples mecanismos asociativos", lo que fundamenta en parte el llamado análisis conductual realizado en escenarios artificiales, el cual es extraído y transpolado a las distintas situaciones educativas.

Retomando la historia, Watson (18781958), fundamentado en los estudios de Pavlov (1849-1936) (apud ROJAS; EGUIBAR, 2001), establece como objeto de estudio de la psicología, el comportamiento; constructo que se puede observar, se puede medir y, según el desarrollo de la teoría de Watson, es manipulable. Así, para Watson, el aprendizaje es entendido como un cambio de comportamientos asociados a estímulos externos; por lo que investiga acerca de cuáles son los estímulos necesarios para lograr ciertos comportamientos en el sujeto (AGUDELO; GUERRERO, 1973).

En esta misma línea ideológica respecto a la forma de concebir la producción del conocimiento psicológico desde una postura similar a la de la física clásica, en la que se manipulan las variables de un sistema delimitado y la búsqueda de corroboración de hipótesis que conlleven a establecer generalidades válidas, se desarrolla la propuesta teórica de Skinner (1904-1990). Para el investigador, la educación se entiende como el proceso mediante el cual el sujeto aprende el cúmulo de conductas que son socialmente correctas, a través del mecanismo de refuerzo por premio-castigo; todo ello sustentado en sus estudios sobre la asociación de estímulos externos apropiados, con conductas deseables en sujetos (AGUDELO; GUERRERO, 1973).

Como se ha visto, la educación orientada desde el empirismo-positivismo-conductismo es reduccionista en cuanto a la forma de concebir los procesos y los roles de los sujetos, pues desde allí se asume la enseñanza como 
un proceso que consiste en proporcionar contenidos o información, es decir, depositar acumulativamente en la mente del alumno aquello que lo capacita o lo califica para estar en sociedad; que es lo que Freire (1970) llamaría educación bancaria. Este enfoque centra el proceso en el docente, lo que deja al sujeto que aprende como un espectador o receptor pasivo frente a un monólogo irrefutable e infalible y fuera de contextos próximos, lo que se ha asociado a la condición de desmotivación hacia el aprendizaje (TAPIA, 2001; CAMPANARIO, 2002; HERNÁNDEZ, 2002), pues el estudiante realmente no comprende la mayor parte de la información que se le expone (WEENK, 1999).

Con los aspectos anteriormente expuestos, es posible describir los elementos característicos de la Didáctica General propia de la llamada Educación Tradicional o el modelo de Transmisión-Recepción-Repetición: a) el sujeto que llega a la escuela no tiene conocimientos o los que tiene no son relevantes para su formación; trae su mente vacía, b) el profesor es el poseedor del conocimiento y deposita el saber en la mente de quienes aprenden, c) es notable la motivación del aprendizaje, a través del mecanismo de refuerzo de conductas por premio-castigo, d) el mecanismo enunciado se traduce en castigos o premios para reforzar la conducta esperada o adecuada, en calificaciones y descalificaciones, en ganar y perder, en hábil e inhábil, en entrenado y no entrenado, o en capacitado y discapacitado.

b. Constructivismo (Glasersfeld, Piaget, Vygotsky y Ausubel):

El constructivismo no solo es una nueva forma de comprender el aprendizaje humano, sino que además abarca aspectos epistemológicos que significan nuevas visiones sobre el conocimiento y así mismo alternativas en la pedagogía y en la didáctica. Así, en términos generales, se puede decir que el constructivismo como paradigma, es el fundamento de varias teorías, pues asume: a) la imposibilidad de observar directamente y con ello surge la negación de establecer qué es lo verdadero, b) en ese sentido, el conocimiento se hace relativo y depende de la perspectiva o forma de ver que tiene quien observa, c) lo que implica que el conocimiento es relativo, histórico $\mathrm{y}$ contextual (social, política, económica $\mathrm{y}$ ambientalmente), d) así mismo, se asume que el conocimiento no es un descubrimiento de la realidad, sino una construcción subjetiva de los sujetos en comunidades; e) y son dichas comunidades quienes acuerdan los criterios de demarcación relativos que determinan qué es lo que corresponde o lo que es válido para su corpus de saber, f) y quienes acuerdan las formas de construir conocimiento, así como el lenguaje y formas de difusión.

El constructivismo como un paradigma opuesto al realismo del positivismo, se fundamenta en dos principios: a) El conocimiento no es recibido pasivamente, sino construido activamente por el sujeto cognoscente; y b) La función de la cognición es adaptativa y sirve a la organización del mundo experiencial, no al descubrimiento de una realidad ontológica. Aceptar sólo el primer principio es considerado como constructivismo trivial (centrado en el proceso cognitivo) por quienes sí aceptan ambos principios comunes en el constructivismo radical (que además del proceso cognitivo, asume el trasfondo ontológico y epistémico) (GLASERSFELD, 1991).

Para el mismo autor (1995), el constructivismo radical va más allá de una negación del conocimiento del mundo exterior, estableciendo una barrera entre el sujeto $\mathrm{y}$ los objetos exteriores y desplazando así al mundo exterior, concepto que prevalecía en el conductismo. En palabras de Noddings (1990) y basado en los fundamentos del constructivismo radical, el conocimiento y la realidad misma son construcciones de la mente, así pues, el desplazamiento, por parte del constructivismo radical, de la fuente de las regularidades que dan lugar al conocimiento, desde el mundo exterior de los realistas al sujeto constructor, es el argumento para la reconstrucción de algunos 
conceptos tradicionales como conocimiento, verdad, comunicación y comprensión (MARTÍNEZ, 1999).

Por otro lado, aunque ya se ha dado la discusión en torno a las diferencias entre las teorías de Piaget (1975) y Vygotsky (1977), por cuanto el primero asume que el desarrollo precede al aprendizaje y da cuenta de prácticamente todas las facetas del desarrollo humano, y por cuanto el segundo plantea que el aprendizaje puede preceder al desarrollo, otros autores asumen una postura hacia la convergencia de las teorias del aprendizaje que pueden ser consideradas como constructivistas. Así mismo, Ausubel \&t Novak \&t Hanesian (1983) postulan el proceso de Aprendizaje Significativo, el cual se da cuando el sujeto logra de manera activa que la nueva información se ancle a los esquemas mentales previos que posee. Dichos esquemas previos son producto de las experiencias del sujeto y si estos no son un soporte suficiente para la nueva información, el aprendizaje no se dará.

Para Rodríguez (1999), existen elementos comunes en las teorías de Piaget y Vygotsky, los cuales aportan al objetivo de establecer los principales aspectos que caracterizarían una Didáctica General Constructivista. Así, para la autora es clave: a) el conocimiento como construcción activa del sujeto (no se hereda, ni se adquiere por transmisión directa), b) la interacción social como mediación de la construcción, y c) las herramientas o andamiaje que hace posible dicha interacción (la escuela es un espacio para ello).

Chadwik (1999) resume que los elementos principales en las teorías de los tres autores son: a) el aprendizaje es una construcción propia del sujeto, la cual se va produciendo por interacción entre disposiciones internas y el medio ambiente, b) el conocimiento no es una copia exacta de la realidad, c) el aprendizaje no es un sencillo proceso de recepción y acumulación de información, sino que es un proceso activo situado en contextos reales, d) la organización del conocimiento en esquemas mentales será lo que facilite aprendizajes posteriores, e) la enseñanza y el currículo se debe fundamentar en la comprensión de dichos esquemas previos, f) el aprendizaje se da por interacción social y está mediado por elementos culturales particulares.

En la actualidad el constructivismo goza de una gran popularidad. En el contexto educativo, por lo menos la palabra y sus connotaciones más suscitadas, el término constructivismo, aparecen repetidamente en numerosas publicaciones, en los pronunciamientos de teóricos e investigadores, así como en planes de estudio de todos los niveles y modalidades (RIG0, 2008).

Finalmente, con las reflexiones realizadas en este título del artículo, es posible identificar algunas características que describen una Didáctica General Constructivista: a) se asume que es posible aprender y que el aprendizaje es una construcción del sujeto, b) los procesos se centran en los sujetos que aprenden, c) el docente $\mathrm{u}$ orientador del proceso no solo aborda aspectos conceptuales de las disciplinas o no solo enseña cosas, sino que también aporta al desarrollo de habilidades y así mismo enseña formas de aprender, d) para lograr el aprendizaje, es relevante conocer los saberes previos que tiene el estudiante, para así orientar el proceso de manera consecuente, e) la evaluación es un proceso que permite regular y promover el aprendizaje, f) es coherente con el constructivismo orientar los aprendizajes a través de la resolución de problemas, el trabajo cooperativo, el trabajo por proyectos, la investigación dirigida, el estudio de casos y la contextualización de los saberes, entre otras posibilidades centradas en el sujeto que aprende, g) el docente u orientador del proceso promueve diversas estrategias que motivan el aprendizaje.

\section{Reflexiones respecto a qué observar sobre lo didáctico en la práctica docente}

Para la investigación en torno al objeto la práctica docente, para este caso asumido desde la dimensión didáctica, surge la cuestión ¿Qué permite evidenciar lo didáctico en la práctica 
docente?, que ha sido planteada en el problema al inicio del artículo como una orientación para hacer operativa la observación del proceso enseñanza-aprendizaje, justifica precisar varios aspectos que se han desarrollado aquí y otros relacionados: a) es posible observar acciones, indagar sobre percepciones, concepciones $\mathrm{y}$ creencias, y así mismo sistematizar experiencias, las cuales permitan construir un modelo que describa el proceso de enseñanza-aprendizaje, b) la interpretación de los registros de la observación es problemática en tanto depende de la postura o limitaciones epistémicas del observador, no sólo en lo investigativo, sino también frente al proceso que se estudia, c) se han identificado la Didáctica General y las Didácticas Específicas como formas de entender el fenómeno enseñanza-aprendizaje, pudiéndose hacer una interpretación de los datos de la investigación desde allí.

Teniendo claros los motivos por los cuales se opta por hacer la descripción-interpretación de lo didáctico desde la Didáctica General y asumiendo el Constructivismo (desde la perspectiva radical, asumiendo lo ontológico, lo epistémico y los procesos cognitivos del sujeto, desde los planteamientos de Glasersfeld (1991 y 1995), explicados en el apartado 3.2 de este artículo) como una opción ampliamente argumentada como pertinente, ahora es tarea concretar cuáles son los elementos que caracterizan la Didáctica General Constructivista y con ello esbozar algunos indicadores para observar acciones, indagar sobre percepciones, concepciones y creencias, y así mismo sistematizar experiencias didácticas en el marco de la práctica docente. Entonces es posible afirmar como aspectos observables que:

\section{El docente tiene en cuenta los conocimientos $\mathrm{y}$ las ideas previas del estudiante, o cita experiencias previas o tareas, o retoma los resultados de la evaluación, para planear y/o re-orientar el desarrollo de los temas de la clase y el currículo en general.}

2. El profesor propone ejemplos, casos, problemas o situaciones para articular o motivar el desarrollo de los temas. Dichas motivaciones no sólo se relacionan con la disciplina a enseñar, sino también con el contexto de los estudiantes; además son posibles de solucionar o resolver con el conocimiento, habilidades y estrategias que se le enseñarán al estudiante en ese periodo académico.

3. El profesor enseña a los estudiantes aspectos como el diseño de actividades, manejo de herramientas, procesamiento de información, gestión de recursos, protocolos, lenguajes especializados, experimentación, estrategias de evaluación o regulación de los procesos y/o demás aspectos prácticos relacionados con la disciplina y la gestión de sus conocimientos y habilidades.

4. El docente promueve las reflexiones éticas o valorativas sobre el conocimiento disciplinar tratado en clase y sobre las implicaciones sociales-ecológicas que su desarrollo y/o aplicación podrían implicar; así como sobre los contextos frente a los cuales articulan o ponen en práctica 0 aplican el conocimiento aprendido.

5. El docente promueve que el estudiante participe en clase y en la reflexión sobre el currículo, que muestre los resultados de sus tareas o actividades en clase y extra-clase, que explique las razones de sus preguntas, que manifieste sus motivaciones, que argumente sus afirmaciones, que proponga alternativas de solución a situaciones y que describa la forma en que realiza los procesos. 6. El docente y los estudiantes discuten el sentido, objetivos, criterios, dinámica y usos de los resultados de la evaluación. La evaluación es un proceso que permite reconocer, regular y planear los aprendizajes. 7. El docente tiene una reflexión, postura e interpretación, más o menos estructurados o más o menos sistematizados, del proceso de construcción de los aprendizajes de los estudiantes y de la estructuración y desarrollo de su práctica docente en lo didáctico.

8. Los aspectos mencionados anteriormente conducen a que la práctica docente sea 
objeto de estudio permanente por parte de quien la orienta; lo que se traduce en que el docente es un investigador en el aula.

De esta manera, los aspectos presentados anteriormente aportan en varios aspectos sobre la investigación sobre la práctica docente: a) establecen un criterio teórico sobre lo que se puede entender como "lo didáctico" en la práctica docente, b) son un soporte para estructurar la metodología a través de la cual se investigará en torno a la práctica docente, c) son un referente desde el cual se puede hacer la interpretación de las evidencias obtenidas sobre la práctica docente en términos didácticos, d) son una alternativa para proponer procesos o intervenciones didácticas, reflexiones en torno a la práctica docente $\mathrm{y} / \mathrm{o}$ procesos de formación de docentes en lo didáctico desde la Didáctica General.

\section{Consideraciones finales}

Como aporte al campo de investigación de la profesionalización de la docencia universitaria, se puede resaltar la actualización que se hace de la relación sujeto-objeto, en tanto se relativiza una vez más la posibilidad de observar directamente la realidad; aspecto que parece tomarse como obvio en muchas investigaciones educativas, pero que no es tomado como un problema epistemológico que conlleva a asumir que lo que se observó (registró, describió, midió, valoró) de un sistema es verdad. Esto es claro para el presente caso, por cuanto se cuestiona respecto a qué es lo que se quiere ver de la práctica docente universitaria y cuáles son las limitaciones del observador en el momento en que pretende ver los aspectos didácticos de clases referidas a disciplinas que no son su disciplina y las cuáles no tienen referentes como la didáctica específica.

Lo anterior se hace evidente cuando los investigadores con formación en psicología, en educación en general, en didáctica de las ciencias, en administración y en sociología, se enfrentan a observar clases de áreas como medicina, práctica médica en clínicas, mercadeo, empresas e inglés como lengua extranjera, entre muchas otras, puesto que el obstáculo epistemológico consiste en que ellos como observadores no tienen formación específica respecto a tales áreas del conocimiento y tampoco existen referentes sobre la didáctica específica para cada una de estas disciplinas. De esta manera, el planteamiento, reflexión y estudio que se presenta en este artículo, es una alternativa ante tal obstáculo, pues con el reconocimiento de los aportes que puede dar el establecimiento de unos criterios generales desde una Didáctica General Constructivista, se puede solventar en parte el problema respecto a qué y cómo ver respecto al objeto de estudio de la práctica docente universitaria, sin por ello dejar de lado la discusión en torno a las didácticas específicas.

Así, como contribución al campo de investigación en práctica docente universitaria se aporta de manera concreta a:

- La reflexión sobre los docentes universitarios que no tienen formación pedagógica; lo que constituye un problema de investigación.

- La identificación del obstáculo epistemológico que tiene el observador-investigador al acercarse y tratar de dar cuenta de prácticas docentes de disciplinas que están fuera de su propia formación profesional.

- La discusión en torno a las didácticas generales y las didácticas específicas; definiendo la primera como una alternativa para orientar la observación de las prácticas docentes universitarias de disciplinas que no poseen didáctica específica.

- La propuesta de los criterios de una Didáctica General Constructivista, los cuales orientan la observación, así como otros posibles instrumentos de investigación. 


\section{Referencias}

ADÚRIZ-BRAVO, Agustín; IZQUIERDO, Mercè. Acerca de la didáctica de las ciencias como disciplina autónoma. Revista Electrónica de Enseñanza de las Ciencias, Vigo, v. 1, n. 3, p. 130-140, 2002. Disponible en: <http://www.saum.uvigo.es/reec/volumenes/ volumen1/Numero3/Art1.pdf>. Acceso en: Feb. 2011.

AGUDELO, Rosa; GUERRERO, Juan. El sistema psicológico de B. F. Skinner. Revista Latinoamericana de Psicología, Bogotá, v. 5, n. 2, p. 191-216, 1973. Disponible en: <http://redalyc.uaemex.mx/redalyc/src/inicio/ArtPdfRed.jsp?iCve=80550206>. Acceso en: Feb. 2011.

AGUIAR, Márcia Angela da S.; MELO, Márcia Maria de Oliveira. Pedagogia e faculdades de educação: vicissitudes e possibilidades da formação pedagógica e docente nas IFES. Educação \& Sociedade, Campinas, v. 26, n. 92, p. 959-982, 2005. Disponible en: <http:// www.scielo.br/scielo.php?script=sci_arttext\&pid=S0101-73302005000300012\&lng=es\&nrm=is0> . Acceso en: Mayo 2012.

AISENBERG, Bestriz. Didáctica de las ciencias sociales: ¿desde qué teorías estudiamos la enseñanza? Revista de Teoría y Didáctica de las Ciencias Sociales, Mérida, n. 3, p. 1-28, 1994. Disponible en: <http://www.saber.ula.ve/bitstream/123456789/23916/1/ bol3_beatriz_aisenberg.pdf>. Acceso en: Feb. 2011.

TAPIAALONSO, J. Motivación y estrategias de aprendizaje. Principios para su mejora en alumnos universitarios. En García Varcárcel Muñoz-Repiso, A. (Coord.) Didáctica Universitaria. Madrid: La muralla, 2001.

AUSUBEL, David; NOVAK, Joseph; HANESIAN, Helen. Psicología Educativa: Un punto de vista Cognoscitivo. México: Trillas, 1983.

CACHAPUZ, Antonio et al.. A emergência da didática das ciências como campo específico de conhecimento. Revista Portuguesa de Educação, Braga, v. 14, n. 1, p. 155-195, 2001. Disponible en: <http://redalyc.uaemex.mx/redalyc/src/inicio/ArtPdfRed. jsp?iCve=37414108>. Acceso en: Feb. 2011.

CAMPANARI0, Juan. ¿Cómo influye la motivación en el aprendizaje de las ciencias? Revista Interuniversitaria de Formación del Profesorado, Zaragoza, n. 33, p. 121-140, 2002. Disponible en: <http://www2.uah.es/imc/webens/127.htm l>. Acceso en: Feb. 2011.

CEBRIÁN, Manuel; VAIN, Pablo. Una mirada acerca del rol docente universitario, desde las prácticas de la enseñanza en entornos no presenciales. Pixel-Bit: Revista de Medios y Educación, Sevilla, n. 32, marzo, p. 117-129, 2008. Disponible en: <http://www. sav.us.es/pixelbit/pixelbit/articulos/n32/8.pdf>. Acceso en: Feb. 2011.

CHADWICK, Clifton. La psicología del aprendizaje desde el enfoque constructivista. Revista Latinoamericana de Psicología, Bogotá, v. 31, n. 003, p. 463-475, 1999. Disponible en: <http://redalyc.uaemex.mx/redalyc/src/inicio/ArtPdfRed.jsp?iCve=80531303>. Acceso en: Feb. 2011.

CHAMLIAN, Helena Coharik. Docência na universidade: professores inovadores na USP. Cadernos de Pesquisa, São Paulo, n. 118, p. 41-64, 2003. Disponible en: <http://www.scielo.br/scielo.php?script=sci_arttext\&pid=S0100-15742003000100003\&ln $\mathrm{g}=\mathrm{es \& nrm}=\mathrm{is} 0>$. Acceso en: Mayo 2012.

DÍAZ, Joaquín. Didáctica de las lenguas extranjeras: los enfoques comunicativos. Didáctica (Lengua y Literatura), Madrid, n. 8, p. 87-103, 1996. Disponible en: <http://www.ucm.es/BUCM/revistas/edu/11300531/articulos/DIDA9696110087A.PDF>. Acceso en: Feb. 2011.

DURKHEIM, Emile. Las reglas del método sociológico. Madrid: Ediciones Morata, 1978.

FERNÁNDEZ, Gabriela. Pedagogía, psicología y didáctica de la matemática. Rect@, Sevilla, actas 8, n. 1, p. 101-116, 2000. Disponible en: <http://150.214.55.100/asepuma/sevilla2000/m3-01.pdf>. Acceso en: Feb. 2011.

FIGUEROA, Neyilse, PÁEZ, Haydee. Pensamiento didáctico del docente universitario. Una perspectiva desde la reflexión sobre su práctica pedagógica. Fundamentos en Humanidades, San Luis, v. 18, n. 2, p. 11-136, 2008. Disponible en: <http://redalyc. uaemex.mx/src/inicio/ArtPdfRed.jsp?iCve=18411970006>. Acceso en: Feb. 2011.

FRANCIS, Susan. Hacia una caracterización del docente universitario "excelente": Una revisión a los aportes de la investigación sobre el desempeño del docente universitario. Educación, San José, v. 30, n. 001, p. 31-49, 2006. Disponible en: <http://redalyc. uaemex.mx/redalyc/src/inicio/ArtPdfRed.jsp?iCve=44030103>. Acceso en: Feb. 2011. 
FREIRE, Paulo. Pedagogía del Oprimido. México: Siglo XXI, 1970.

GALLEGO-BADILLO, Rómulo. Un concepto epistemológico de modelo para la didáctica de las ciencias experimentales. Revista Electrónica de Enseñanza de las Ciencias, Vigo, v. 3, n. 3, p. 301-319, 2004. Disponible en: <http://www.saum.uvigo.es/reec/ volumenes/volumen3/Numero3/ART4_VOL3_N3.pdf>. Acceso en: Feb. 2011.

GLASERSFELD, Ernst Von. Constructivism in Education. In: LEWY, A. The International Encyclopedia of Curriculum. Oxford: Pergamon Press, 1991.

Radical Constructivism: A way of knowing and learning. Londres: The Falmer Press, 1995.

GODINO, Juan. Perspectiva de la didáctica de las matemáticas como disciplina tecnocientífica. Granada: Universidad de Granada, 2010. Disponible en: <http://www.ugr.es/ jgodino/fundamentos_teoricos/perspectiva_ddm.pdf>. Acceso en: Feb. 2011.

Indicadores de la idoneidad didáctica de procesos de enseñanza y aprendizaje de las matemáticas. XIII Conferencia Interamericana de Educación Matemática. Recife: Comité Interamericano de Educación Matemática, 2011. Disponible en: $<$ http://www.ugr.es/ jgodino/eos/jdgodino_indicadores_idoneidad.pdf>. Acceso en: Feb. 2011.

HERNÁNDEZ, Ana. Los desafíos de la docencia universitaria. Educación, San José, v. 26, n. 002, p. 117-124, 2002. Disponible en: $<$ http://redalyc.uaemex.mx/redalyc/src/inicio/ArtPdfRed.jsp?iCve=44026212>. Acceso en: Feb. 2011.

MARTínEZ, Alberto. Constructivismo radical, marco teórico de investigación y enseñanza de las ciencias. Enseñanza de las ciencias, Barcelona, v. 17, n. 3, p. 493-502, 1999. Disponible en: <http://ddd.uab.cat/pub/edlc/02124521v17n3p493.pdf>. Acceso en: Feb. 2011.

MENNA, S; SALVATICO, L. Racionalidad y metodología en el Novum Organum de Bacon. Cuadernos de la Facultad de Humanidades y Ciencias Sociales, San Salvador de Jujuy, n. 015, p. 139-144, 2002. Disponible en: <http://redalyc.uaemex. mx/redalyc/src/inicio/ArtPdfRed.jsp?iCve=18501513>. Acceso en: Feb. 2011.

MONTENEGRO, Helena; FUENTEALBA, Rodrigo. El formador de futuros profesionales: una forma de comprender la docencia en la educación superior universitaria. Calidad en la Educación, Osorno, n. 32, p. 235-251. 2010. Disponible en:<http://www.cned.cl/ public/secciones/seccionpublicaciones/doc/67/cse_articulo907.pdf> Acceso en: Mayo 2012.

MONTERO, Patricio. Desafíos para la profesionalización del nuevo rol docente universitario. Ensaio: Avaliação e Políticas Públicas em Educação, Rio de Janeiro, v. 15, n. 56, p. 341-350, 2007. Disponible en: <http://www.scielo.br/scielo.php?script=sci_ artext\&pid=S0104-40362007000300003\&lng=es\&nrm=iso>. Acceso en: Mayo 2012.

MORENO, María. De la matemática formal a la matemática escolar. PNA, Andalucía, v. 1, n. 3, p. 99-111, 2007. Disponible en: <http://www.pna.es/Numeros2/pdf/Moreno2007De.pdf>. Acceso en: Feb. 2011.

NODDINGS, N. Constructivism in Mathematics Education. In: DAVIS, R.B.; MAHER, C.A; NODDINGS, N. (Eds.). Constructivist Views on the Teaching and Learning of Mathematics. Reston: National Council of Teachers of Mathematics, p. 7-18, 1990.

NÚÑEZ, María; FERNÁNDEZ, Eduardo; ROMERO, Antonio. Research in language didactics: a model for the evaluation of oral discursive competence in secondary education. Porta Linguarum, Granada, v. 9, enero, p. 111-126, 2008. Disponible en: <http:// www.ugr.es/ portalin/articulos/PL_numero9/8\%20Pilar\%20Nunez.pdf>. Acceso en: Feb. 2011.

OLIVEIRA, Cláudia Chueire; VASCONCELLOS, Maura Maria Morita. A formação pedagógica institucional para a docência na Educação Superior. In: Interface (Botucatu), Botucatu, v. 15, n. 39, p. 1011-1024, 2011. Disponible en: <http://www.scielo.br/ scielo.php?script=sci_arttext\&pid=S1414-32832011000400005\&lng=es\&nrm=iso>. Acceso en: Mayo 2012.

OCDE. Revisión de Políticas Nacionales de Educación: La Educación Superior en Chile. Chile: Organización para el Desarrollo y la Cooperación Económicas y el BIRD/Banco Mundial. 2009.

PAGÉS, Joan. La didáctica de las ciencias sociales, el currículum y la formación del profesorado. Signos teoría y práctica de la educación, Gijón, año 5, n. 13, p. 38-51, 1994. Disponible en: <http://www.quadernsdigitals.net/datos_web/hemeroteca/r_3/ nr_39/a_617/617.html>. Acceso en: Feb. 2011. 
PIAGET, Jean. Introducción a la epistemología genética. Buenos Aires: Paidós, 1975.

POZO, Juan. Teorías cognitivas del aprendizaje. Barcelona: Morata, 1989.

PRATS, Joaquim. Disciplinas e interdisciplinariedad: el espacio relacional y polivalente de los contenidos de la didáctica de las ciencias sociales. Iber: Didáctica de las ciencias sociales, geografía e historia, Barcelona, n. 24, p. 7-18, 2000.

Líneas de investigación en didáctica de las ciencias sociales. História \& Ensino Revista do Laboratório de Ensino de Históriória/UEL, Londrina, v. 9, p. 1-25, 2003. Disponible en: <http://www.ub.edu/histodidactica/CCSS/Lineas-Prats.pdf>. Acceso en: Feb. 2011.

PRETTO, Nelson De Luca; ROCHA, Nicia Cristina. A formação continuada de professores universitários e as tecnologias digitais. Educar em Revista, Curitiba, n. 37, p. 153-169, 2010. Disponible en: <http://www.scielo.br/scielo.php?script=sci_ arttext\&pid=S0104-40602010000200010\&lng=es\&nrm=iso > . Acceso en: Mayo 2012.

REIS, Michele Xavier dos; EUFRASIO, Daniela Aparecida; BAZON, Fernanda Vilhena Mafra. A formação do professor para o ensino superior: prática docente com alunos com deficiência visual. Educar em Revista, Belo Horizonte, v. 26, n. 1, p. 111-130, 2010. Disponible en: <http://www.scielo.br/scielo.php?script=sci_arttext\&pid=S0102-46982010000100006\&lng=es\&nrm=i so>. Acceso en: Mayo 2012.

RIGO, Marco. Constructivismo educativo, actividad y evaluación del docente: relato de algunas posibles incongruencias. Reencuentro, Xochimilco, v. 53, diciembre, p. 125-134, 2008. Disponible en: <http://redalyc.uaemex.mx/pdf/340/34005311. pdf>. Acceso en: Feb. 2011.

RIOLFI, Claudia Rosa; ALAMINOS, Cláudia. Os pontos de virada na formação do professor universitário: um estudo sobre 0 mecanismo da identificação. Educação e Pesquisa, São Paulo, v. 33, n. 2, p. 297-310, 2007. Disponible en: <http://www. scielo.br/scielo.php?script=sci_arttext\&pid=S1517-97022007000200008\&lng=es\&nrm=iso>. Acceso en: Mayo 2012.

RODRÍGUEZ, Wanda. El legado de Vygotski y de Piaget a la educación. Revista Latinoamericana de Psicología, Bogotá, v. 31, n. 003, p. 477-489, 1999. Disponible en: <http://redalyc.uaemex.mx/src/inicio/ArtPdfRed.jsp?iCve=80531304>. Acceso en: Feb. 2011.

ROJAS, Gerardo; EGUIBAR, José. Pavlov y los reflejos condicionados. Elementos: Ciencia y cultura, Puebla, v. 8, n. 41, p. 49-54, 2001. Disponible en: <http://redalyc.uaemex.mx/src/inicio/ArtPdfRed.jsp?iCve=29404107>. Acceso en: Feb. 2011.

SHULMAN, Lee. Knowledge and teaching. Foundations of the new reform. Harvard Education Review, Cambridge, 57(1), p. 1-22, 1987.

. Taking Learning Seriously. Change: The Magazine of Higher Learning, Philadelphia, 31(4), p. 10-17, 1999.

TISKI, Sergio. Introdução à questão da filosofia primeira em Comte. Acta Scientiarum. Human and Social Sciences, Maringá, n. 32, v. 2, p. 217-222, 2010. Disponible en: <http://periodicos.uem.br/ojs/index.php/ActaSciHumanSocSci/article/ view/9369/9369>. Acceso en: Feb. 2011.

TOVAR-GÁLVEZ, Julio. Modelo metacognitivo como integrador de estrategias de enseñanza y estrategias de aprendizaje de las ciencias, y su relación con las competencias. Revista Iberoamericana de Educación, Madrid, v. 46, n. 7, p. 1-9, 2008. Disponible en: <http://www.rieoei.org/deloslectores/2161Tovarv2.pdf>. Acceso en: Feb. 2011.

Propuesta de modelo de evaluación multidimensional de los aprendizajes en ciencias naturales y su relación con la estructura de la didáctica de las ciencias. Revista Eureka Sobre Enseñanza y Divulgación de las Ciencias, Cádiz, v. 5, n. 3, p. 259-273, 2008. Disponible en: <http://www.apac-eureka.org/revista/Volumen5/Numero_5_3/Tovar_2008.pdf>. Acceso en: Feb. 2011.

. Propuesta para el aprendizaje del concepto reacción química y generación de actitudes hacia la ciencia, a través del estudio de los problemas de la organización espacial del laboratorio y el manejo de residuos químicos. Revista Electrónica de Enseñanza de las Ciencias, Vigo, v. 8, n. 2, p. 490-504, 2009. Disponible en:<http://www.saum.uvigo.es/reec/volumenes/ volumen8/ART6_Vol8_N2.pdf. >. Acceso en: Feb. de 2011.

TRUJILLO, Fernando. En torno a la interculturalidad: reflexiones sobre cultura y comunicación para la didáctica de la lengua. Porta Linguarum, Granada, v. 4, junio, p. 23-39, 2005. Disponible en: <http://www.ugr.es/ portalin/articulos/PL_numero4/trujillo.pdf>. Acceso en: Feb. 2011. 
VEZ, José. La DLE: de hoy para mañana. Porta Linguarum, Granada, v. 1, enero, p. 5-30, 2004. Disponible en: <http://www.ugr. es/ portalin/articulos/PL_numero1/vez.pdf>. Acceso en: Feb. 2011.

VYGOTSKY, Lev. Pensamiento y lenguaje. Buenos Aires: La Pléyade, 1977.

WEENK, G. Learning Pyramid. Enschede: Educational Center, University of Twente, 1999.

ZAMBRANO, Armando. Conocimiento, saber y pensamiento: una aproximación a la didáctica de las matemáticas. Educere, Mérida, año 8, n. 26, julio/septiembre, p. 407-413, 2004. Disponible en: <http://www.saber.ula.ve/bitstream/123456789/19898/2/ articul015.pdf>. Acceso en: Feb. 2011.

ZANÓN, Javier. Psicolingüística y didáctica de las lenguas: Una aproximación histórica y conceptual. MarcoELE: Revista de Didáctica, Ribarroja del Turia, v. 5, p. 1-30, 2007. Disponible en: <http://marcoele.com/descargas/5/zanon-psicolinguistica.pdf>. Acceso en: Feb. 2011.

Recebido en: 21.12.2011

Aprobado en: 02.07.2012

Julio César Tovar-Gálvez es magíster en docencia de química, docente-investigador en temas como educación en la complejidad, formación de docentes, didáctica de las ciencias, procesos metacognitivos y educación ambiental en la Facultad de Educación, Universidad Antonio Nariño (Bogotá-Colombia).

Germán Antonio García Contreras es magíster en docencia de química, docente e investigador en temas como formación en competencias, didáctica de las ciencias y formación de docentes de ciencias en la Facultad de Ciencias Básicas, Universidad Antonio Nariño (Bogotá-Colombia). E-mail: germangarcia7@hotmail.com. 